\title{
Mutational signatures shift induced by chemotherapeutic agents, 5-Fluorouracil and Oxaliplatin, in the gut microbiome
}

Fei Su

Beijing Hospital https://orcid.org/0000-0003-4737-4651

Zhao Liu

Affiliated Hospital of Xuzhou Medical University

Lihui Zou

Beijing Hospital

Xue Xu

Beijing Hospital

Qingfeng Luo

Beijing Hospital

Hexin Li

Beijing Hospital

Wenzhuo Jia

Beijing Hospital

Min Tang

Beijing Hospital

Yingying Huang

Beijing Hospital

Yunbo Zhao

Beijing Hospital

Lin Li

Beijing Hospital

Bin Ai

Beijing Hospital

Meng Chen

Chinese Academy of Medical Sciences and Peking Union Medical College

Jie Ma

Beijing Hospital

Gang Zhao

Bejing Hospital

Hongtao Xu 
Beijing Hospital

Fei Xiao ( $\square$ xiaofei3965@bjhmoh.cn )

https://orcid.org/0000-0003-2054-901X

\section{Research}

Keywords: Chemotherapeutic agents, 5-Fluorouracil, Oxaliplatin, gut microbiota

Posted Date: January 7th, 2020

DOI: https://doi.org/10.21203/rs.2.20234/v1

License: (c) (i) This work is licensed under a Creative Commons Attribution 4.0 International License. Read Full License 


\section{Abstract}

We developed a powerful framework for taxonomy composition and genomic variation analysis to investigate the mutagenesis effect and proliferation influence of chemotherapeutic agents, such as 5Fluorouracil (5-FU) and Oxaliplatin (Oxi) on gut microbiota. Using the gut microbiome data of 68 time serial stool samples, we detected 1.45 million variations among the chemotherapy groups and found the drugs significantly affected mutation signatures of gut microbiota. About 786 faecal metagenomes of 755 individuals from 5 different cohorts were analyzed to build the mutation pattern of gut microbiota from health samples. Oxi notably increase transversion rate, while 5-FU reduced the rate. We also performed in vitro experiments to confirm that chemotherapeutic agents could disrupt the pattern of genetic variant in the intestinal microorganisms. Post-chemotherapy samples had specific gut microbiome signatures with higher abundance of Bacilli and a lack of anaerobic bacteria. In addition, drug-associated functional alterations were also found: metabolism changes in the 5-FU group implied that gut microbiota could provide additional NAD + to inhibit cancer cell autophagy; in the Oxi group, the ribosome and lysine biosynthesis genes were obviously enriched. According to molecular evolution analysis, traits related to protein secretion system showed evidence of strong selection pressure from the drugs, which could be a novel potential treatment strategy for chemotherapy-induced diarrhea. Our study provides a blueprint for characterizing the role of microbes and drug-microbe interaction in the gut microbiota response to chemotherapy.

\section{Introduction}

Chemotherapeutics have long been used to treat a variety of human tumors [1]. As one of the commonly used chemotherapy protocols, the FOLFOX regimen contains different chemotherapeutic agents (e.g. 5Fluorouracil (5-FU) and Oxaliplatin (Oxi)) with a broad range of cytotoxicity [2]. These drugs can effectively inhibit DNA replication of cancer cells via different mechanisms of action. For example, 5-FU produces FdUMP, which directly inhibits thymidylate synthase and thus causes thymine-less cell death [3]. In addition, the products of 5-FU may be converted into FUTP or FdUTP, leading to RNA or DNA damage, respectively. An understanding of the interaction of chemotherapeutics and gut microbial populations will probably trigger applications towards improve the effectiveness of chemotherapy.

Chemotherapeutic agents may affect gut microbial communities by disrupting the homeostatic balance among resident microorganisms; meanwhile, they can accelerate the microbe evolution at molecular level [4-6]. Many recent studies have explored the interaction between microbiota and anticancer drugs along with interventions aimed at shaping microbiota to optimize drug efficacy and reduce side effects [7]. However, previous studies mainly focused on the taxonomic characterization and functional compositions of different cohorts at the genus or species level [8]. Variation in taxonomic abundance as well as functions encoded by these gut microbiota have been described in the cohorts of inflammatory bowel disease (IBD) [9], type 2 diabetic (T2D) [10], hypertension [11] and liver cirrhosis [12]. However, genomic variations within species, which leads to their phenotypic diversity and adaptations to chemotherapeutic agents, have only been studied in a few taxa [13]. For example, in the common gut 
commensal bacteria Escherichia coli [14] just few point mutations can confer clinically relevant antibiotic resistance, and the natural variation in a single gene can lead to pathogenic adaptation.

Given the importance of the gut microbiota in human health and a growing number of studies reporting associations between gut microbiota and diseases [15], a better understanding of the interaction of gut microbiota with chemotherapeutic drugs during chemotherapy help to improve the efficacy of the anticancer chemotherapeutic agents. We recruited 37 cancer patients and collected pre- and postchemotherapy stool samples to study the taxonomic composition, metabolic capacity and molecular evolution during the chemotherapy. In order to avoid the bias caused by distinct diet, regional and genetic differences, we collected healthy samples from well-known studies about Han Chinese with the standard clinical assessments and same sequence platform to build the basic of microbiome structure and mutation pattern. We analyzed our cohort and stool samples from T2D [10], liver cirrhosis [12], and hypertension cohorts [11] with the same bioinformatics pipeline and parameters. Our goal was to build a power framework for metagenomics analysis to gather basic knowledge on the genomic variation landscape and taxonomic abundance shift in gut metagenomes during chemotherapy.

\section{Results}

Overall composition of gut microbiota during chemotherapy

To characterize the diversity and composition of gut microbiome during the chemotherapy, we characterized the gut microbial composition of 68 samples from 37 patients (6 samples were excluded due to DNA extraction failure) using $16 \mathrm{~S}$ rRNA gene sequencing. Detail information about stool samples and patients were listed in the Supplementary Table S1 and S2. This cohort consisted of 7 patients using 5-FU, 13 patients using 5-FU + Oxi, and 17 patients using Oxi. All the participants were from a cohort study in Beijing Hospital. Using samples collected at the start of chemotherapy (Day0) and 30 days later (Day30), we traced the dynamic changes of gut microbial species by ecological alpha and beta diversity measures. The chemotherapeutic drugs caused modest changes in the gut microbiome. Shannon diversity and Chao1 index were calculated to estimate the within-sample (a) diversity (Fig. 1A) and there is no significant alteration pattern. Weighted UniFrac principal coordinate analysis was used to compare community phylogenetic composition among samples and the result revealed a separation between Day0 and Day30 along axis 1 (explaining $41.5 \%$ of the variation in the data, Supplementary Figure S1). Beta diversity represents how much the community changed in comparison to the baseline (Day0) and was calculated with Weighted UniFrac distance between Day0 and Day30 (Supplementary Table S3). ANOSIM with permutations confirmed significant separation of samples ( $P$-value $=0.02, r=0.24)$. No significant differences in microbial communities were observed when the $0 x i$ and 5 -Fu + Oxi groups were analyzed separately. Although the adjusted P-value (adjusted P-value $=0.06$ ) is not significant, it indicated that there was a difference pattern in the microbial composition of Day0 and Day30 with 5-Fu. Next, we used LDA effect size (LEfSe) to analyze the microbial communities of different chemotherapeutic drugs. For all Day30 samples, we identified gut microbiome signatures with higher abundance of Bacilli (specifically Streptococcaceae and Lactobacillaceae, Fig. 1B). For 5-Fu, seven 
species were discovered as biomarkers for separating gut microbiota between Day0 and Day30 (Supplementary Figure S2). Five of these species were higher, and two were lower in the Day0 than Day30. For example, the abundances of Bacteroides coprocola and Streptococcus anginosus were lower in Day30 (P-value $=0.03$, two-sided Wilcoxon signed-rank test). Contrary to 5-FU, in the Oxi group, there were 8 of these species were higher and on was lower in the Day0 than Day30. No significant change was observed in the 5-FU + Oxi group. To gain further insights into the interaction between chemotherapeutic drugs and gut microbiota, we used the same gut microbiota DNA preparations for independent Illumina shotgun sequencing. The yielded sequences belonged to 10 phyla and 381 species, including several DNA viruses (Supplementary Table S4 and Figure S3). Firmicutes was the most abundant phylum, accounting for $40.9 \pm 22.3 \%$ of the total reads, followed by Bacteroidetes ( $36.3 \pm 23.7 \%$ ), Proteobacteria $(15.7 \pm 23.4 \%)$ similar to previous studies [12]. Two commensal species including S. salivarius and L. salivarius were significant enriched after the chemotherapy. (Supplementary Figure S4) Correlation tests between the abundance estimates for bacterial taxa at genus level were performed by using the two methods (Pearson correlation coefficient: 0.84; P-value <2.2 × 10-16; Fig. $1 \mathrm{C}$ and Supplementary Figure S5) highlighted a highly positive correlation between $16 \mathrm{~S}$ rRNA and shotgun metagenomics sequencing.

Functional alterations in gut microbiota during the chemotherapy

To determine the functional alterations with the chemotherapeutic agents, we use the HUMAnN2/MinPath [16] and Kyoto Encyclopedia of Genes and Genomes (KEGG) database [17] to evaluate gut microbial functions across groups in our study cohort. According to the HUMAnN2/STAMP analysis of the metabolic function pathways [18], most differences occurred in carbohydrate metabolism and energy related pathways in the 5-FU group (Fig. 2). The microbiome from Day30 included more genes involved in nicotinamide adenine dinucleotide (NAD) salvage pathway (PYRIDNUCSAL-PWY) than that from Day0 in the 5-FU group (Adjust P-value $=1.4 \times 10^{-4}$ ). Previous study suggested an active role for the NAD salvage pathway in modulating cancer cell viability via the replenishing of the NAD reservoir [19], which is a novel strategy to protect cell against DNA damage. Moreover, amino acid biosynthesis, such as L-phenylalanine and arginine, UDP-N-acetyl-D-glucosamine biosynthesis pathway were more active in the samples in the 5-FU group. In other two groups, few pathways were found to be significantly different before and after chemotherapy (Supplementary Figure S6).

In order to deeply explore the functional role of the gut microbiota during the chemotherapy, all the genes were aligned to the KEGG database and the abundance of KEGG Ortholog groups (KOs) and pathways for each sample was estimated using the method similar to RPKM [20]. About 277 KOs were significantly enriched among the three groups (P-value $<0.05$, two-side Wilcoxon rank sum test; Supplementary Table S5). Overrepresentation of 3 KEGG pathways involved in metabolism was observed in the 5 -FU group with hypergeometric distribution, which involved in the categories "Galactose metabolism" and "Phosphotransferase system (PTS)" (Supplementary Table S6). Enriched genes encoding PTS, which reflected increased representation of multiple carbohydrate transporters, was an important mechanism to confer resistance to selection pressure, such as antibiotic, as reported in the previous studies [21]. In the Oxi group, two KEGG pathways "Ribosome" and "Lysine biosynthesis" were significantly enriched. 
Although Oxi, a platinum-based drug, is generally considered to be DNA-damaging agents, recent studies have unexpectedly shown that Oxi do not alter DNA integrity but instead inhibit rDNA transcription, leading to p53 induction, most likely through the "impaired ribosome biogenesis checkpoint" [22].

Genomic variation in intestinal microorganisms

To enable comparative analyses in multiple metagenomes and to identify the mutation signatures in the different groups, we modified the previous method [23] to identify single nucleotide polymorphisms (SNPs), short insertions / deletions (InDels, range $1 \mathrm{bp}$ to $50 \mathrm{bp}$ ) and structural variations (more than $50 \mathrm{bp}$ ) in each sample. First, we used 3,934 prokaryotic genomes to generate a set of reference genomes (Supplementary Table S8) for the analysis of genomic variation in gut microbial species in 786 samples. Next, local realignment around high-quality InDels, which is a frequently used strategy in genomic variation analysis, was performed to correct mapping errors to improve the accuracy of variant calling [24]. We only considered variants with allele frequency larger than $1 \%$ and supported by more than 5 reads. To validate our SNP calling procedure, we used the approaches to calculate error rates in 40 essential single-copy marker genes, following the method mentioned previous [23]. False-positive rates were estimated at 3.6\% (Supplementary Table S9).

We identified 1.45 million SNPs from 343 genomes (at least 3 SNPs for one genome), of which 1.38 million SNPs $(95 \%)$ in 47 genomes $(0.88 \%$ of the total $156 \mathrm{Mb})$ across 68 stool samples from 37 subjects (Supplementary Figure S7). We also identified 26,662 indels and 56,820 structural variants. Subsequent analyses were restricted to SNPs due to their orders of magnitude higher count over other variation types. Examination of the SNP distributions of the protein coding genes of the selected 47 gut bacteria revealed that 4189 genes with at least one non-synonumous mutation in the 47 microbe species had valid coverage ( $\geq 10 \mathrm{X}$ depth) with sufficient prevalence (Supplementary Table S10). Among them, we identified 22 genes $(0.5 \%)$ with significantly differentiated SNP densities between Day0 and Day30 samples (Wilcoxon signed-rank test, P-value < 0.05$)$. Most of these genes $(17 / 22)$ were found in Bacteroides thetaiotaomicron VPI-5482, the second most common human commensal bacteria, whose reference genome contains 4779 protein-coding genes [25] (Supplementary Table S11).

Then, we checked the substitution pattern shift between Day0 and Day30. A large portion of base substitutions were attributable to $C>T$ substitution, which ranged from $29.5 \%$ to $43.0 \%$ in Day0, and from $35.0 \%$ to $42.3 \%$ in Day30. Comparisons of the mutation rates in other Chinese cohorts, including liver cirrhosis [12], hypertension [11] and T2D [10] (Fig. 3 and Supplementary Table S12) showed a significantly reduced transversion (Student's $T$ test, adjust P-value $=0.0003$ ) and transition rate (Student's T test, adjusted P-value $=0.01$ ) the 5-FU group (Supplementary Table S13). By contrast, we found an increased ratio of transversion (Student's T test, adjusted P-value $=5.4 \times 10^{-4}$ ) and transition (Student's T test, adjusted P-value $=8.2 \times 10^{-7}$ ) in the group of Oxi. The similar mutation pattern was also observed the studies about the effect of antibiotic on the genome [26]. Thus, we conclude that genome-wide substitutions rates of gut microbiota were influenced by chemotherapeutic drugs. To directly test whether selection might have biased the mutation rate of different regions in the genomes, we examined the 
synonymous and nonsynonymous mutation from coding region from all cohorts. Nonsynonymous / synonymous mutation ratio from Day30 were not significantly different from Day0 (Student's T test, Pvalue $>0.05$ in all comparison), indicating that the vast majority of acquired amino acid-altering mutations were not selectively promoted by chemotherapeutic agents but simply accumulated in a neutral fashion.

In order to validate the shift of mutation pattern, we explored how stool-isolated microorganism mutation rates change in vitro when treated with 5-FU and Oxi. We exposed four gut species from four different patients (two Escherichia coli strains, Citrobacter sp. MGH104 and Enterococcus faecium), all of which were with significantly low growth rate limited by the chemotherapeutic agents, in different concentrations of $75 \mu \mathrm{M}$ 5-FU and Oxi (Supplementary Figure S8). Most notably, the transition/transversion ratio of mutations do covary with agents concentrations (Supplementary Figure S9).

Dn/Ds across gut species and individuals

To gain further insights on the molecular mechanisms driving the functional diversification of the gut microbiota, the gene families identified in the assembled metagenome were annotated based on the KEGG and we calculated, for each KO, the ratio between the number of nonsynonymous (Dn) and synonymous (Ds) changes, a proxy for evolutionary pressure (Supplementary Figure S10). Our analyses showed that the average Dn/Ds was 0.063 the median was 0.016 from $939 \mathrm{KO}$ (Supplementary Table S14 and Figure S10). About $17.9 \%$ (17.6\% in the 5 -FU group, $17.7 \%$ in the $5-\mathrm{FU}+$ Oxi group, and $18.3 \%$ in the Oxi group) of the gene families had significantly higher Dn values and lower Ds values than the mean value calculated over all annotated sequences (one-side Fisher test, FDR $<0.05$ ), suggesting that they may be under positive selection (Supplementary Table S15 and Figure S11). A closer investigation of these gene families revealed that positive selection signatures markedly characterize diverse proteins involved in the cell division, as well as proteins essential for amino acid biosynthesis (Fig. 4 and Supplementary Figure S12). According to the abundance analysis of KO items between Day 0 and Day30, we found genes were under significant positive selection pressure, such as secY, which is a key in the protein secretion system and important for critical cell functions, like pathogens and virulence. In addition, as in the previous study [27], we also found the coding sequence of phage infection protein (yhgE) and genes related to transport system were under positive selection. Thus, the genes under positive selection might have a key role in the interaction between bacteria and chemotherapeutic drugs and provide the bacteria additional survival advantage during the chemotherapy.

\section{Discussion}

The human gut microbiota is highly complex and exists in a dynamic balance between symbiosis and pathogenesis, which can influence almost any aspect of host physiology [28]. Growing evidence suggests that the gut microbiota not only plays a key role in carcinogenesis but also influences the efficacy and toxicity of anticancer therapy $[29,30]$. The microbiota modulates the host response to chemotherapy via 
numerous mechanisms, such as alteration of community structure and immune microenvironment. Furthermore, exploitation of the microbiota offers opportunities for the personalization of chemotherapeutic regimens and the development of novel therapies.

Chemotherapeutic agents, Oxaliplatin and 5-FU, exert their cytotoxic effect mostly through DNA damage. When DNA damage is caused by chemotherapeutic drugs, the microbiota composition also changes, which can further affect drug efficacy and overall health. It's very important to systematic explore the interaction between chemotherapeutic drugs and gut microbiota not only from microbiome composition $[31,32]$ but also genomic variation $[23,33]$. In this study, we first used two different methods (16S rRNA and shotgun metagenomics sequencing) to investigate the disruption of the intestinal microbiome in terms of taxonomic composition. Correlation tests between the abundance estimates for bacterial taxa show that the two methods highlighted a highly positive correlation. According to the 16S rRNA sequence, we found that different chemotherapeutic agents had distinct effect on the gut microbiota. 5-FUassociated bacteriome shifts included depletion of common health-associated commensals from the genera Streptococcus and Bacteroides and enrichment of Gram-negative bacteria such as Clostridium hathewayi and Lachnospiraceae bacterium. Previous study has shown that 5-FU could improve T celldependent antitumor immunity [34] and Clostridia strains play a key role in enhancing $T_{\text {reg }}$ cell abundance and inducing important anti-inflammatory molecules, such as interleukin-10 [35]. In contrast, Oxi-associated bacteriome showed different shift patterns including depletion of commensals from the genus Lachnospiraceae noname and enrichment of genus Lactobacillus and Streptococcus.

Subsequently, we use shotgun metagenomics with a quantitative metagenomic species approach to identify which species significantly change during the chemotherapy with different agents. Two commensal species including S. salivarius and L. salivarius were significant enriched after the chemotherapy. According to the previous studies, these two species appeared to be highly resistant to 5FU [36], which maybe the main driver for a community dysbiosis. We hypothesized that if 5-FU was responsible for the dysbiosis changes seen during chemotherapy, then depleted taxa should be susceptible to the drug while the enriched species would be resistant to 5 -FU. The resultant damage to the intestinal barrier increases the risk of colitis, bacterial translocation and infection. Probiotic supplementation may have extra beneficial effects to correct dysbiosis of gut microbiota after the chemotherapy.

Concomitant with the alteration of gut microbial composition, we also observed a dysbiosis in bacterial gene functions and different chemotherapeutic agent showed distinct influence patterns. Microbial metabolism may cause side effects severe enough to necessitate cessation of chemotherapy. The metagenome of 5 -FU patients was enriched in genes associated with NAD salvage pathway. Since NAD ${ }^{+}$ plays central roles in a variety of biological processes ranging from cellular metabolism and energy production in both human and microbiology. So, it is reasonable to hypothesis that gut microbiota could provide additional $\mathrm{NAD}^{+}$to inhibit cancer cell autophagy and enhance survival of cancer cells [19]. By contrast, the metagenome of Oxi patients were enriched in genes associated with Ribosome. Oxi, unlike cisplatin and carboplatin, kills cancer cells not only through the DNA-damage response, but also by 
inducing ribosome biogenesis stress [37]. In order to adapt the selection pressure from Oxi, only the species with high copy number of genes related to ribosome can survive during the chemotherapy similar to cancer cells, which may be why genes involved with ribosomes were significantly enriched.

Next, in order the study the effective of DNA damage caused by chemotherapeutic agents, we collected the metagenomics sequencing data of 768 stool samples from 755 Chinese human individuals with the standard clinical assessments and re-analyzed the shift of mutation pattern with unified bioinformatics pipeline. Consistent to our expectation, we found obvious shift in the mutation signatures. A significantly reduced transversion rate (G:C->T:A) were observed in the 5-FU group. In contrast, we found an increased ratio of transversion in the Oxi group. What's more, the genome-wide substitutions rates of gut microbiota were influenced by chemotherapeutic drugs without bias among different genomic regions. To further confirm that the shift of mutation signatures were due to strong and diverse environmental selection, we analyzed the patterns of correlation between gene function and the Dn/Ds ratio across all the KO modules. The stable Dn/Ds ratios of most KEGG modules (80.1\%) between Day0 and Day30 suggest that the core microbiome were evolutionarily conserved. However, there are some modules, such as genes involved with cell division, protein export and phage-related show high level selection pressure. A previous study has shown that phage-related genes usually have fast mutation rate and are under positive selection [38]. However, the other genes, such as zapA and mutT, which are housekeeping genes involved in cell amplification and previously are thought to be with slow rate of amino acid evolution, have remarkably high Dn/Ds ratio and are under positive selection pressure. On the other hand, in order to adaptive to pressure of chemotherapeutic agents, bacteria take different strategies to obtain the survival advantage during the therapy, for example by secreting an amount of protein to modify the host microenvironment with Sec system, which was a potential chemotherapeutic target to many human pathogen.

\section{Conclusions}

We have described the disordered profiles of gut microbiota in cancer patients treated with different chemotherapeutic agents, explored the mutation pattern of different drugs, and provided a new clue for the interaction between drugs and microbe. Our findings suggest that a distinct intestinal microbiome pattern of gut dysbiosis during the chemotherapy is dominated by Bacilli and a lack of other bacteria and the potential restorative influence of probiotic supplements should be investigated in the chemotherapeutic research. Positive selection on the protein export system provide additional survival advantage for gut microbiota and could be a potential therapy target for gut dysbiosis.

\section{Methods \& Materials}

Cohort recruitment and sample collection

Participants were recruited in the Oncology Department of Beijing Hospital, Beijing, China, in 2018. This study was approved by the Beijing Hospital Ethics Committee. Written informed consent was obtained 
from all patients. The exclusion criteria included: with a history of inflammatory bowel diseases; having been exposed to probiotics, prebiotics or broad-spectrum antibiotics within 30 days; or having received nasal-tube feeding or parenteral nutrition in the month prior to initiation of the study. Detail information about all patients were listed in the Supplementary Table S1. We collected two faecal samples from each participant. A faecal sample was collected on hospital in-patient admission (Day0), prior to administration of chemotherapy, and 30 days later immediately prior to chemotherapy (Day30). For each faecal sample, one gram of stool was collected into a sterile tube and then subsequently stored at $-80^{\circ} \mathrm{C}$ for molecular analysis. Total genomic DNA was extracted from $200-500 \mathrm{mg}$ of fecal samples using a QIAamp DNA Stool Mini kit (Qiagen, Germany) according to the manufacturer's instructions. The concentration of the extracted DNA was measured by a NanoDrop2000 (Thermo Scientific, USA), and then the DNA was stored at $-80^{\circ} \mathrm{C}$.

16 S ribosomal RNA sequencing and bioinformatics analysis

To develop the 16S rRNA amplicon libraries, the V3-V4 region of 16S rRNA gene was PCR-amplified with the primers 341F (5'-CCTAYGGGRBGCASCAG-3') and 806R (5'-GGACTACNNGGGTATCTAAT-3'), modified by adding barcodes for multiplexing [39]. Pooled amplicons were paired-end sequenced (PE $2 \times 250 \mathrm{bp}$ ) by using an Illumina HiSeq 2500 platform according to the manufacture's protocol. Paired-end reads were merged and quality filtered using FLASH and QIIME [40]. Chimeras were detected and removed against the Gold reference database using the UCHIME algorithm [41]. Sequences with $\geq 97 \%$ similarity were assigned to the same OTUs. For each representative sequence, the GreenGenes Database was used based on RDP classifier algorithm to annotate. Alpha diversity analysis including Shannon and Chao1 were calculated with QIIME. Phylogenetic beta diversity distances, including unweighted and weighted UniFrac distances were measured using QIIME. Principal-Component Analysis (PCoA) was performed to visualize by ggplot2 package in $\mathrm{R}$ software (Version 3.5.0). Further, diversity analyses, such as Adonis and Anosim, were performed by running a workflow on QIIME.

Shotgun metagenomics sequencing library construction

For shotgun metagenomics sequencing, adaptive focused acoustics (Covaris) was used to shear a standard volume of $50 \mu \mathrm{l}$ of the extracted DNA. DNA libraries were prepared using Illumina TruSeq Sample Preparation Kit (Illumina, USA) according to the manufacturer's protocol. Libraries were quantified using an Agilent Bioanalyzer 2100 (Agilent Technologies, USA). Paired-end sequencing (PE $2 \times$ $150 \mathrm{bp}$ ) was performed on successful DNA libraries using an Illumina HiSeq X-Ten instrument at the Annoroad Genome Biotech at Beijing. Finally, we obtained 4.27 GB to 8.9 GB of raw data for each sample (average 5.98 GB, Supplementary Table S2).

Metagenomics bioinformatics analysis

Concatenated and human DNA sequences were removed using KneadData with default parameter (http://huttenhower.sph.harvard.edu/kneaddata). The remaining high-quality reads were assembled with IDBA-UD (Version: 2.04, parameters: -pre_correction --min_contig 200) [42]. We mapped the clean reads 
against scaffolds using Bowtie2 (Version: 2.3.4.2) [43]. Genes (minimum length of 100 nucleotides) were predicted on contigs longer than $500 \mathrm{bp}$ and annotated using Prokka (Parameters: --metagenome) [44]. The gene abundance was determined by using the method similar to RPKM (reads per kilo bases per million reads) used for quantifying gene expression from RNA sequencing data. In brief, high-quality reads were counted with $H T S e q-c o u n t ~[45]$. For each gene, $G_{i}$, the number of reads that aligned to it divided by the length of the gene was calculated as $N G_{i}$ and the relative abundance $R N G_{i}$, of each gene in each sample (n genes) was computed using the following formula:

Species-level quantitative taxonomic profiling was performed using MetaPhlAn2 (Version:

9760413b180f) on the KneadData-filtered reads [46]. Community composition was calculated with MetaPhlan2 using the default settings. Taxonomic profiles included bacteria, archaea, microbial Eukaryotes, and viruses were inferred by MetaPhIAn2 using the $1 \mathrm{M}$ unique clade-specific marker genes identified from 17,000 reference genomes (13,500 bacterial and archaeal, 3,500 viral, and 110 eukaryotic).

Functional annotation

Two different methods were used for functional alteration and pathway composition analysis. First, pathway-level composition was calculated with HUMANn2 using the UniRef90 database with default settings, which was followed by further statistical analysis and visualization in STAMP [18]. In order to obtain the detail information of each metabolic pathway, all genes in our genomes were aligned to the KEGG database using DIAMOND (Version 0.7.9.58, parameter: -k 50 -sensitive -e 0.00001) and KOBAS [47]. Each protein was assigned to the KEGG Orthology (KO) families by the highest scoring annotated hits containing at least one HSP scoring over 60 bits. The abundance of KEGG orthology / module was calculated using the methods as mentioned above. For KEGG orthology enrichment analysis, significant enriched KEGG pathway was defined as the adjusted P-value $(<0.05)$ of the hypergeometric test as the previous study [48].

Generation of a reference genome set

We followed the previous method to build the reference genomes to study the genomic variation of gut microbiota [23]. About 3934 prokaryotic genomes were downloaded from NCBI

(ftp://ftp.ncbi.nlm.nih.gov/genomes/refseq/bacteria) on August 1 2018. A set of 40 universal single copy marker genes was identified in these genomes using HMM profiles made for each marker gene from the corresponding orthologous group from eggnog database $[49,50]$. For each marker gene, pairwise DNA sequence identities between all genomes were calculated using BLASTn (Version 2.2.25, parameter: evalue $<10^{-5}-\mathrm{F} \mathrm{F}$ ) [51]. For each genome pair, the median identity of all marker genes was used as a proxy for average nucleotide identity (ANI) between the two genomes. Using an operational 95\% ANI recommended for identifying species, we generated 929 clusters of genomes. In order to select a reference genome from each cluster, high quality reads from a subset of shotgun metagenomes (bgi-BGI06A, bgi-DLF001, bgi-DOF002 from T2B cohort, nHF611710, nHF411719 from HTN, HD-2, HD-31 from 
liver, 1-1, 4-1, $5-1$ from this study) were mapped to the 3934 genomes using Bowtie2 with the options "--very-fast". Then, the genome with the highest read coverage was selected resulting in a set of 958 reference genomes, each likely representing a unique species (see Supplementary Table S8)

Mapping to reference genomes and variant calling

Illumina reads from 718 fecal samples, including the T2D, Liver and Hypertension cohorts were quality controlled using a KneadData. KneadData-filtered reads were mapping to reference genomes using Bowite2 (Version: 2.3.4.2) with default parameter. The number of reads mapping to reference genomes were counted and normalized by the genome size in order to obtain quantitative relative abundances of each genome in every sample.

To improve the accuracy of genomic variation, we modified the protocol of previous study [23]. First, the SAMtools/BCFtools suite was used for calling high quality InDel supported by more than 5 reads and at least one read on each strand [52]. We then performed local realignment of the aligned reads with those high-quality InDel using the Genome Analysis Toolkit (GATK) [53] to minimize the mismatch bases. GATK's HaplotypeCaller was used to call variants with default parameter. BreakDancer was used to detect the structural variation with at least three reads supported the event [54].

Positive selection analysis

CDSs were annotated based on their protein family membership using KOBAS [47]. With MUSCLE [55], a multiple sequence alignment (MSA) of the protein sequences was created using each KEGG Orthology. Based on the MSA and the CDS nucleotide sequences, a codon-based alignment was constructed for each KO module with PAL2NAL using default parameters [56]. We then used FastTree, a relaxed neighbour joining algorithm, to reconstruct a phylogenetic tree for each protein family from the obtained MSA [57]. We excluded low-confidence positions in the alignment with a large number of gaps with Gblocks [58]. Dn/Ds was calculated with PAML [59]. A one-sided Fisher's test was performed to identify protein families with a significant enrichment of Dn versus Ds changes in comparison to the entire sample. The false discovery rate (FDR) was controlled using the Benjamini and Hochberg procedure and alpha set to $5 \%$.

in vitro experiment to validate the mutation pattern shift

A filter-sterilized stock solution of $100 \mathrm{mM}$ 5-FU, Oxi and 5-FU + Oxi (1:1) (Sigma Aldrich) were prepared in DMSO and further diluted to 50,75 and $100 \mathrm{mM}$. Stock solutions were further diluted (1:1000) in culture medium for the experiments. Four mono-isolated gut microorganism were obtained from the stools of recruited patients. LB agar plates used for passaging were prepared by adding stock 5-FU, Oxi and 5-FU + Oxi. Subsequently, cultures were transferred into fresh plates and allowed to grow $48 \mathrm{~h}$ for each batch. After 20 batches, single colony on each plate were then scraped off and transferred to Eppendorf tubes with liquid LB culture and cultured overnight at $37^{\circ} \mathrm{C}$. Total DNA was then extracted using the QIAamp UCP Pathogen Mini Kit (Qiagen, German). The concentration of the extracted DNA was measured by a 
NanoDrop2000 (Thermo Scientific, USA). Illumina DNA sequence libraries were prepared as the mentioned above. JSpeciesWS was used to assign taxonomy of each isolated strains [60]. Reads were then mapped to the closest reference genomes with BWA and variant calling was performed as mentioned above.

Statistical analysis

The Wilcoxon signed-rank test was performed to evaluate differences in richness and diversity for KEGG and microbial taxa at various taxonomic ranks. Mutation rate comparisons were conducted to determine statistical significance between two groups for incidences where differences were declared significant at P-value $<0.05$ with the Student's T-test.

Data Availability

All sequences generated in this study are available in the NCBI sequence read archive under the accession numbers PRJNA551354.

\section{Declarations}

\section{Ethics approval and consent to participate}

This study was approved by the Beijing Hospital Ethics Committee.

\section{Consent for publication}

Yes

\section{Availability of data and material}

All sequences generated in this study are available in the NCBI sequence read archive under the accession numbers PRJNA551354.

\section{Funding}

This work was supported by the National Key Research and Development Program of China (Grant 2018YFC2000500 \& 2017YFC0840102), CAMS Innovation Fund for Medical Sciences (2018-I2M-1-002), National Natural Science Foundation of China (Grant 81571384 \& 81602321 \& 81902618), the Applied Research Program of Capital Clinical Features (Grant Z18110001718172).

\section{Acknowledgments}




\section{Authors' contributions}

F.X. and G.Z. conceived the study and designed the experiments; F.S. and Z.L. performed the experiments; H.T.X. and D.K.C. made clinical samples validation; M.T., Y.Z., L.L., B.A. collected clinical samples and analyzed the clinical data; F.S. analyzed the high throughput sequencing data; F.S. and F.X. wrote the manuscript; and all authors read and approved the final manuscript.

\section{Competing interests}

The authors declare no competing interests.

\section{References}

1. Chabner BA, Roberts TG, Jr.: Timeline: Chemotherapy and the war on cancer. Nat Rev Cancer 2005, $5(1): 65-72$.

2. de Gramont A, Figer A, Seymour M, Homerin M, Hmissi A, Cassidy J, Boni C, Cortes-Funes H, Cervantes A, Freyer $\mathrm{G}$ et al: Leucovorin and fluorouracil with or without oxaliplatin as first-line treatment in advanced colorectal cancer. J Clin Oncol 2000, 18(16):2938-2947.

3. Pinedo HM, Peters GF: Fluorouracil: biochemistry and pharmacology. J Clin Oncol 1988, 6(10):16531664.

4. Baym M, Lieberman TD, Kelsic ED, Chait R, Gross R, Yelin I, Kishony R: Spatiotemporal microbial evolution on antibiotic landscapes. Science 2016, 353(6304):1147-1151.

5. Montassier E, Gastinne T, Vangay P, Al-Ghalith GA, Bruley des Varannes S, Massart S, Moreau P, Potel $\mathrm{G}$, de La Cochetiere MF, Batard $\mathrm{E}$ et al: Chemotherapy-driven dysbiosis in the intestinal microbiome. Aliment Pharmacol Ther 2015, 42(5):515-528.

6. Hong BY, Sobue T, Choquette L, Dupuy AK, Thompson A, Burleson JA, Salner AL, Schauer PK, Joshi P, Fox $\mathrm{E}$ et al: Chemotherapy-induced oral mucositis is associated with detrimental bacterial dysbiosis. Microbiome 2019, 7(1):66.

7. Daillere R, Vetizou M, Waldschmitt N, Yamazaki T, Isnard C, Poirier-Colame V, Duong CPM, Flament C, Lepage P, Roberti MP et al: Enterococcus hirae and Barnesiella intestinihominis facilitate Cyclophosphamide-induced therapeutic immunomodulatory effects. Immunity 2016, 45(4):931-943.

8. Lloyd-Price J, Mahurkar A, Rahnavard G, Crabtree J, Orvis J, Hall AB, Brady A, Creasy HH, McCracken C, Giglio MG et al: Strains, functions and dynamics in the expanded Human Microbiome Project. Nature 2017, 550(7674):61-66.

9. Halfvarson J, Brislawn CJ, Lamendella R, Vazquez-Baeza Y, Walters WA, Bramer LM, D'Amato M, Bonfiglio F, McDonald D, Gonzalez A et al: Dynamics of the human gut microbiome in inflammatory 
bowel disease. Nat Microbiol 2017, 2:17004.

10. Qin J, Li Y, Cai Z, Li S, Zhu J, Zhang F, Liang S, Zhang W, Guan Y, Shen D et al: A metagenome-wide association study of gut microbiota in type 2 diabetes. Nature 2012, 490(7418):55-60.

11. Li J, Zhao F, Wang Y, Chen J, Tao J, Tian G, Wu S, Liu W, Cui Q, Geng B et al: Gut microbiota dysbiosis contributes to the development of hypertension. Microbiome 2017, 5(1):14.

12. Qin N, Yang F, Li A, Prifti E, Chen Y, Shao L, Guo J, Le Chatelier E, Yao J, Wu L et al: Alterations of the human gut microbiome in liver cirrhosis. Nature 2014, 513(7516):59-64.

13. Morowitz MJ, Denef VJ, Costello EK, Thomas BC, Poroyko V, Relman DA, Banfield JF: Strain-resolved community genomic analysis of gut microbial colonization in a premature infant. Proceedings of the National Academy of Sciences of the United States of America 2011, 108(3):1128-1133.

14. Bagel S, Hullen V, Wiedemann B, Heisig P: Impact of gyrA and parC mutations on quinolone resistance, doubling time, and supercoiling degree of Escherichia coli. Antimicrob Agents Chemother 1999, 43(4):868-875.

15. Hauser PJ, VanGordon SB, Seavey J, Sofinowski TM, Ramadan M, Abdullah S, Buffington CA, Hurst RE: Abnormalities in Expression of Structural, Barrier and Differentiation Related Proteins, and Chondroitin Sulfate in Feline and Human Interstitial Cystitis. J Urol 2015, 194(2):571-577.

16. Franzosa EA, Mclver LJ, Rahnavard G, Thompson LR, Schirmer M, Weingart G, Lipson KS, Knight R, Caporaso JG, Segata $\mathrm{N}$ et al: Species-level functional profiling of metagenomes and metatranscriptomes. Nature methods 2018, 15(11):962-968.

17. Kanehisa M, Furumichi M, Tanabe M, Sato Y, Morishima K: KEGG: new perspectives on genomes, pathways, diseases and drugs. Nucleic acids research 2017, 45(D1):D353-d361.

18. Parks DH, Tyson GW, Hugenholtz P, Beiko RG: STAMP: statistical analysis of taxonomic and functional profiles. Bioinformatics 2014, 30(21):3123-3124.

19. Sharif T, Ahn DG, Liu RZ, Pringle E, Martell E, Dai C, Nunokawa A, Kwak M, Clements D, Murphy JP et al: The NAD(+) salvage pathway modulates cancer cell viability via p73. Cell Death Differ 2016, 23(4):669-680.

20. Hu Y, Yang X, Qin J, Lu N, Cheng G, Wu N, Pan Y, Li J, Zhu L, Wang X et al: Metagenome-wide analysis of antibiotic resistance genes in a large cohort of human gut microbiota. Nature communications 2013, 4:2151.

21. Stogios PJ, Cox G, Spanogiannopoulos P, Pillon MC, Waglechner N, Skarina T, Koteva K, Guarne A, Savchenko A, Wright GD: Rifampin phosphotransferase is an unusual antibiotic resistance kinase. Nature communications 2016, 7:11343.

22. Pelletier $\mathrm{J}$, Thomas $\mathrm{G}$, Volarevic S: Ribosome biogenesis in cancer: new players and therapeutic avenues. Nat Rev Cancer 2018, 18(1):51-63.

23. Schloissnig S, Arumugam M, Sunagawa S, Mitreva M, Tap J, Zhu A, Waller A, Mende DR, Kultima JR, Martin J et al: Genomic variation landscape of the human gut microbiome. Nature 2013, 493(7430):45-50. 
24. DePristo MA, Banks E, Poplin R, Garimella KV, Maguire JR, Hartl C, Philippakis AA, del Angel G, Rivas MA, Hanna $M$ et al: A framework for variation discovery and genotyping using next-generation DNA sequencing data. Nat Genet 2011, 43(5):491-498.

25. Xu J, Bjursell MK, Himrod J, Deng S, Carmichael LK, Chiang HC, Hooper LV, Gordon JI: A genomic view of the human-Bacteroides thetaiotaomicron symbiosis. Science 2003, 299(5615):2074-2076.

26. Long H, Miller SF, Strauss C, Zhao C, Cheng L, Ye Z, Griffin K, Te R, Lee H, Chen CC et al: Antibiotic treatment enhances the genome-wide mutation rate of target cells. Proceedings of the National Academy of Sciences of the United States of America 2016, 113(18):E2498-2505.

27. Bulgarelli D, Garrido-Oter R, Munch PC, Weiman A, Droge J, Pan Y, McHardy AC, Schulze-Lefert P: Structure and function of the bacterial root microbiota in wild and domesticated barley. Cell host \& microbe 2015, 17(3):392-403.

28. Sommer F, Backhed F: The gut microbiota - masters of host development and physiology. Nature reviews Microbiology 2013, 11(4):227-238.

29. Panebianco C, Andriulli A, Pazienza V: Pharmacomicrobiomics: exploiting the drug-microbiota interactions in anticancer therapies. Microbiome 2018, 6(1):92.

30. Louis P, Hold GL, Flint HJ: The gut microbiota, bacterial metabolites and colorectal cancer. Nature reviews Microbiology 2014, 12(10):661-672.

31. Hakim H, Dallas R, Wolf J, Tang L, Schultz-Cherry S, Darling V, Johnson C, Karlsson EA, Chang TC, Jeha S et al: Gut Microbiome Composition Predicts Infection Risk During Chemotherapy in Children With Acute Lymphoblastic Leukemia. Clinical infectious diseases : an official publication of the Infectious Diseases Society of America 2018, 67(4):541-548.

32. Yu T, Guo F, Yu Y, Sun T, Ma D, Han J, Qian Y, Kryczek I, Sun D, Nagarsheth $N$ et al: Fusobacterium nucleatum Promotes Chemoresistance to Colorectal Cancer by Modulating Autophagy. Cel/ 2017, 170(3):548-563 e516.

33. Zeevi D, Korem T, Godneva A, Bar N, Kurilshikov A, Lotan-Pompan M, Weinberger A, Fu J, Wijmenga C, Zhernakova $\mathrm{A}$ et al: Structural variation in the gut microbiome associates with host health. Nature 2019.

34. Vincent J, Mignot G, Chalmin F, Ladoire S, Bruchard M, Chevriaux A, Martin F, Apetoh L, Rebe C, Ghiringhelli F: 5-Fluorouracil selectively kills tumor-associated myeloid-derived suppressor cells resulting in enhanced T cell-dependent antitumor immunity. Cancer Res 2010, 70(8):3052-3061.

35. Atarashi K, Tanoue T, Oshima K, Suda W, Nagano Y, Nishikawa H, Fukuda S, Saito T, Narushima S, Hase $\mathrm{K}$ et al: Treg induction by a rationally selected mixture of Clostridia strains from the human microbiota. Nature 2013, 500(7461):232-236.

36. Vanlancker E, Vanhoecke B, Smet R, Props R, Van de Wiele T: 5-Fluorouracil sensitivity varies among oral micro-organisms. J Med Microbio/ 2016, 65(8):775-783.

37. Bruno PM, Liu Y, Park GY, Murai J, Koch CE, Eisen TJ, Pritchard JR, Pommier Y, Lippard SJ, Hemann MT: A subset of platinum-containing chemotherapeutic agents kills cells by inducing ribosome biogenesis stress. Nat Med 2017, 23(4):461-471. 
38. Petersen L, Bollback JP, Dimmic M, Hubisz M, Nielsen R: Genes under positive selection in Escherichia coli. Genome research 2007, 17(9):1336-1343.

39. Caporaso JG, Lauber CL, Walters WA, Berg-Lyons D, Huntley J, Fierer N, Owens SM, Betley J, Fraser L, Bauer $\mathrm{M}$ et al: Ultra-high-throughput microbial community analysis on the Illumina HiSeq and MiSeq platforms. The ISME journal 2012, 6(8):1621-1624.

40. Bokulich NA, Subramanian S, Faith JJ, Gevers D, Gordon JI, Knight R, Mills DA, Caporaso JG: Qualityfiltering vastly improves diversity estimates from Illumina amplicon sequencing. Nature methods 2013, 10(1):57-59.

41. Edgar RC: UPARSE: highly accurate OTU sequences from microbial amplicon reads. Nature methods 2013, 10(10):996-998.

42. Peng Y, Leung HC, Yiu SM, Chin FY: IDBA-UD: a de novo assembler for single-cell and metagenomic sequencing data with highly uneven depth. Bioinformatics 2012, 28(11):1420-1428.

43. Langmead B, Salzberg SL: Fast gapped-read alignment with Bowtie 2. Nature methods 2012, 9(4):357-359.

44. Seemann T: Prokka: rapid prokaryotic genome annotation. Bioinformatics 2014, 30(14):2068-2069.

45. Anders S, Pyl PT, Huber W: HTSeq: a Python framework to work with high-throughput sequencing data. Bioinformatics 2015, 31(2):166-169.

46. Truong DT, Franzosa EA, Tickle TL, Scholz M, Weingart G, Pasolli E, Tett A, Huttenhower C, Segata N: MetaPhIAn2 for enhanced metagenomic taxonomic profiling. Nature methods 2015, 12(10):902-903.

47. Xie C, Mao X, Huang J, Ding Y, Wu J, Dong S, Kong L, Gao G, Li CY, Wei L: KOBAS 2.0: a web server for annotation and identification of enriched pathways and diseases. Nucleic acids research 2011, 39(Web Server issue):W316-322.

48. Jiang Y, Xiong X, Danska J, Parkinson J: Metatranscriptomic analysis of diverse microbial communities reveals core metabolic pathways and microbiome-specific functionality. Microbiome 2016, 4:2.

49. Sorek R, Zhu Y, Creevey CJ, Francino MP, Bork P, Rubin EM: Genome-wide experimental determination of barriers to horizontal gene transfer. Science 2007, 318(5855):1449-1452.

50. Ciccarelli FD, Doerks T, von Mering C, Creevey CJ, Snel B, Bork P: Toward automatic reconstruction of a highly resolved tree of life. Science 2006, 311(5765):1283-1287.

51. Altschul SF, Gish W, Miller W, Myers EW, Lipman DJ: Basic local alignment search tool. Journal of molecular biology 1990, 215(3):403-410.

52. Li H, Handsaker B, Wysoker A, Fennell T, Ruan J, Homer N, Marth G, Abecasis G, Durbin R: The Sequence Alignment/Map format and SAMtools. Bioinformatics 2009, 25(16):2078-2079.

53. McKenna A, Hanna M, Banks E, Sivachenko A, Cibulskis K, Kernytsky A, Garimella K, Altshuler D, Gabriel S, Daly M et al: The Genome Analysis Toolkit: a MapReduce framework for analyzing nextgeneration DNA sequencing data. Genome research 2010, 20(9):1297-1303. 
54. Fan X, Abbott TE, Larson D, Chen K: BreakDancer: Identification of Genomic Structural Variation from Paired-End Read Mapping. Curr Protoc Bioinformatics 2014, 45:15.16.11-11.

55. Edgar RC: MUSCLE: multiple sequence alignment with high accuracy and high throughput. Nucleic acids research 2004, 32(5):1792-1797.

56. Suyama $M$, Torrents $D$, Bork P: PAL2NAL: robust conversion of protein sequence alignments into the corresponding codon alignments. Nucleic acids research 2006, 34(Web Server issue):W609-612.

57. Price MN, Dehal PS, Arkin AP: FastTree: computing large minimum evolution trees with profiles instead of a distance matrix. Mol Biol Evol 2009, 26(7):1641-1650.

58. Castresana J: Selection of conserved blocks from multiple alignments for their use in phylogenetic analysis. Mol Biol Evol 2000, 17(4):540-552.

59. Yang Z: PAML 4: phylogenetic analysis by maximum likelihood. Mol Biol Evol 2007, 24(8):15861591.

60. Richter M, Rossello-Mora R, Oliver Glockner F, Peplies J: JSpeciesWS: a web server for prokaryotic species circumscription based on pairwise genome comparison. Bioinformatics 2016, 32(6):929-931.

\section{Figures}


A.
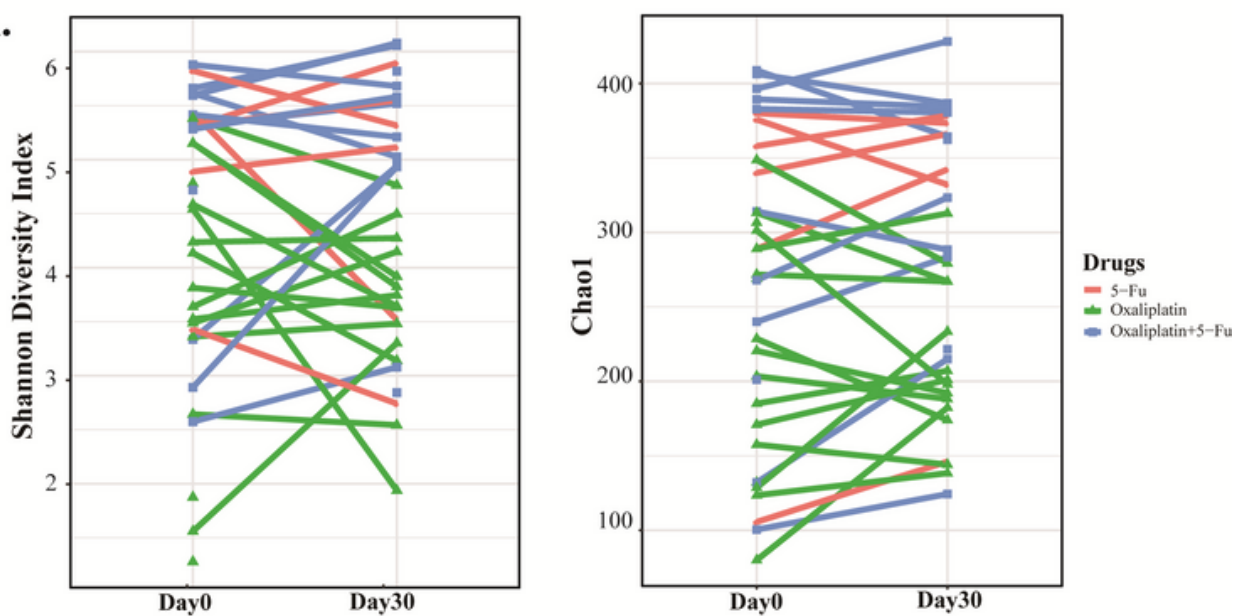

B.

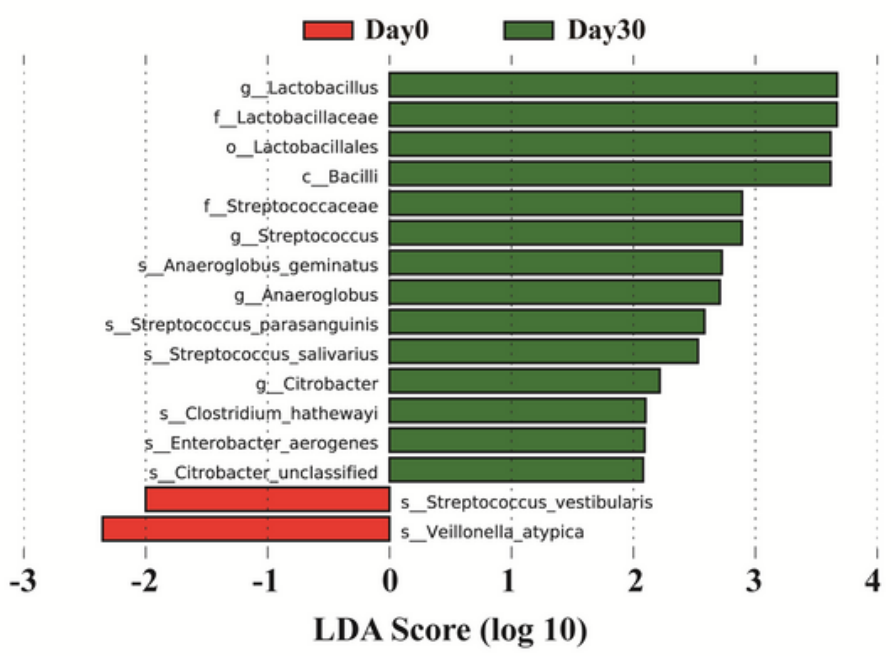

C.

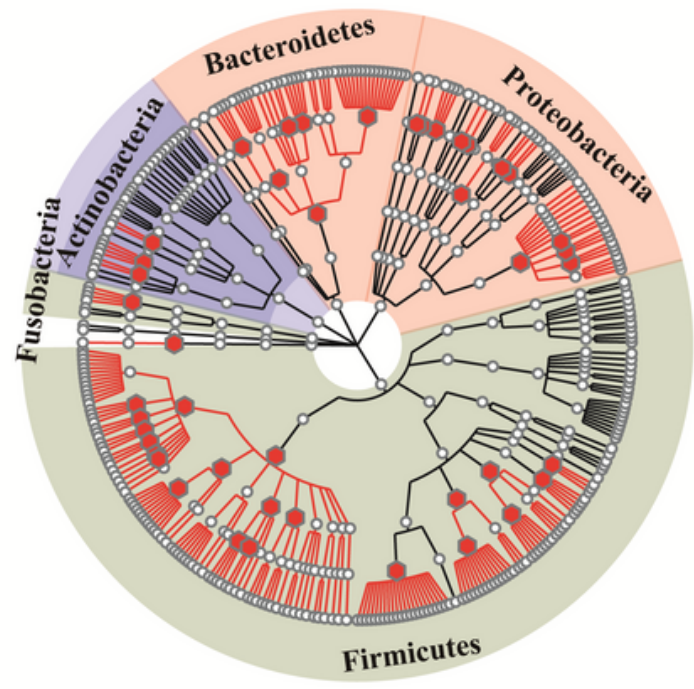

Figure 1

Gut microbial diversity before and after the chemotherapy. A. Shannon diversity (left) and Chao (right) based on 16s rRNA sequencing. Different colors stand for drugs: red for 5-Fluorouracil, green for Oxaliplatin and blue for 5-Fluorouracil and Oxaliplatin. The Y-axis stands for the collection time. B. Cladogram derived from LEfSe analysis of metagenomic sequences based on the shotgun sequencing compared Day0 with Day30. Green shaded areas indicate microbe orders that more consistently describe 
the fecal microbiome from Day30; red shaded areas indicate microbe orders that more consistently describe from Day0. The prefixes " $c$ ", " $o$ ", " $f$ ", " $g$ " and "s" represent the annotated level of class, order, family, genus and species. C. Comparison of 16S rRNA and Metagenome Abundances The tree represents all taxonomically classified species from the shotgun metagenome survey as well as $16 \mathrm{~S}$ rRNA sequence. The branches of the tree do not reflect evolutionary distances. The position of the dots in the tree corresponds to the taxonomic placement of the representative sequences in the NCBI taxonomy. Empty dots represent the phylotypes found in the shotgun metagenome classification, red dots were identified from both methods. 


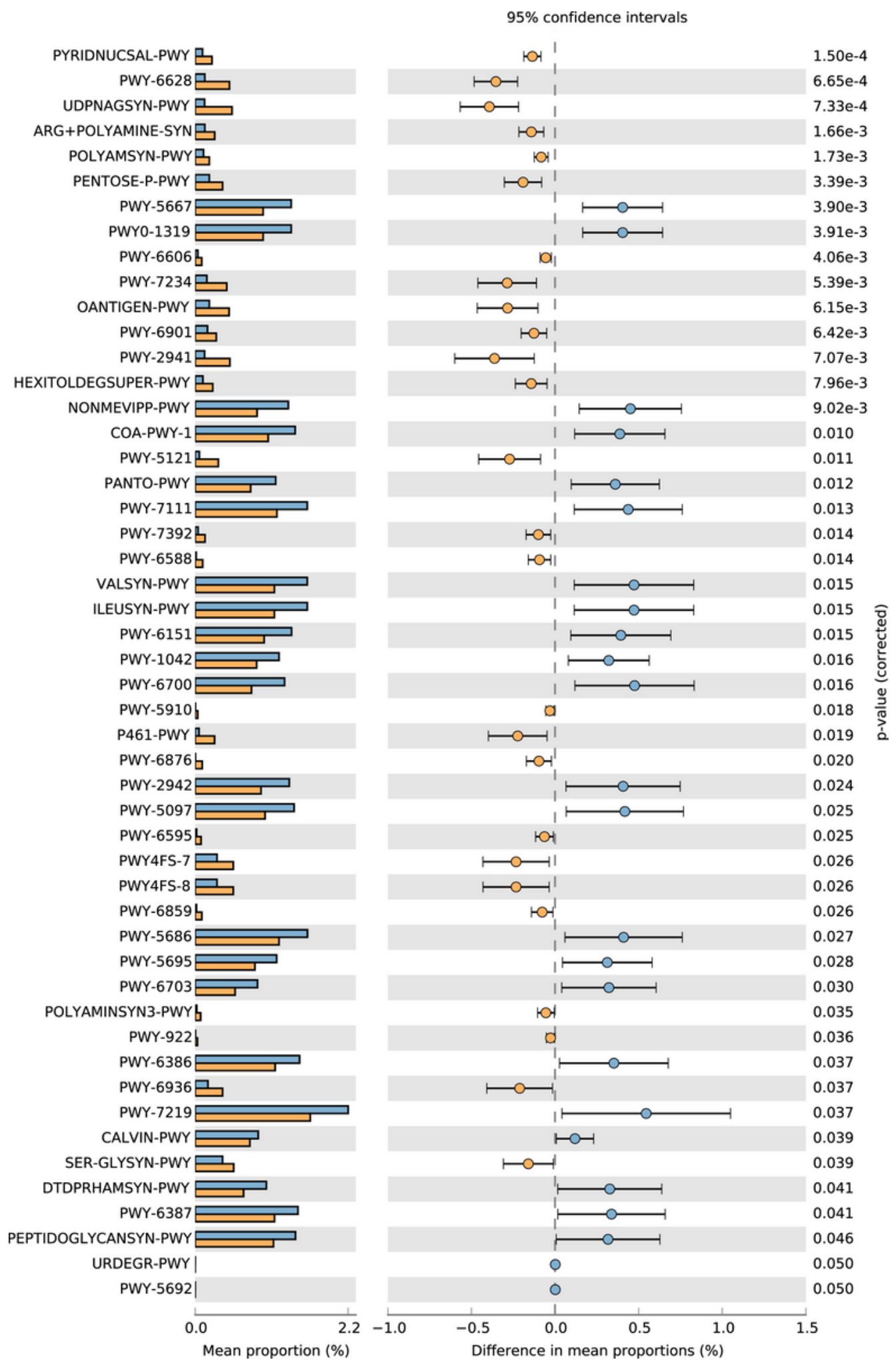

\section{Figure 2}

Extended error bar plots showing the abundance of pathways differing significantly between Day 0 and Day30 in the 5-FU group Extended error bar plots showing the abundance of pathways differing significantly between Day0 and Day30 in the 5-FU group. Corrected P-values are shown on the right. Adjusted P-value $<0.05$ was considered significant. The yellow color stands for Day30 and the blue for Day0. 


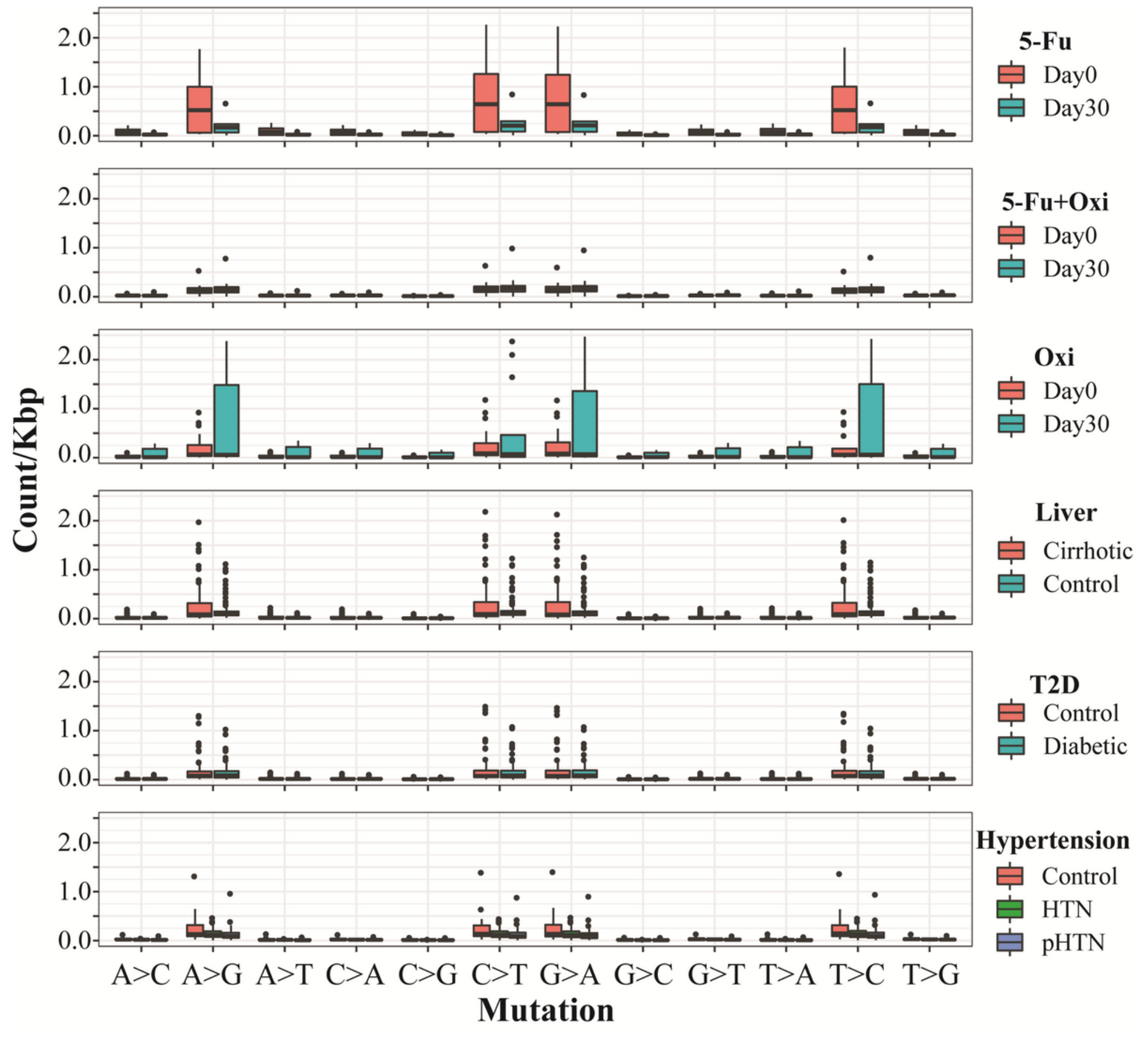

Figure 3

Mutational signatures found in all fecal samples Each cohorts are displayed according to the 12 substitution. The different groups are displayed in different colors as the legends show. The mutation types are on the horizontal axes, whereas vertical axes depict the number of mutations detected in the kilobase attributed to a specific mutation type. 

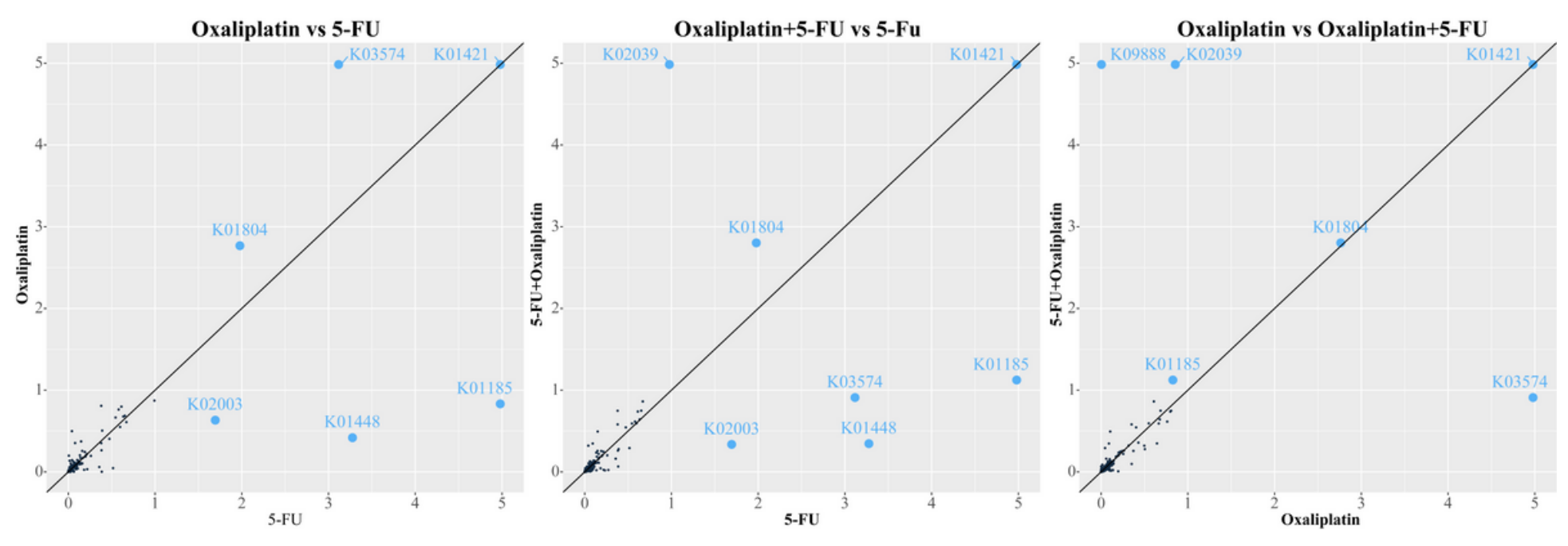

Figure 4

KEGG modules under positive selection in different chemotherapeutic drugs The correlation of average $\mathrm{Dn} / \mathrm{Ds}$ ratio of all KO modules between the drugs. KO modules with average $\mathrm{Dn} / \mathrm{Ds}$ ratio larger than 1 were marked by blue points. Maximum ratio was limited at 5 .

\section{Supplementary Files}

This is a list of supplementary files associated with this preprint. Click to download.

- supplementaryfigures.docx

- supplementarytables.xlsx 